\title{
On the circulation and the position of the forward stagnation point on airfoils
}

\author{
J. Meseguer (corresponding author), G. Alonso, A. Sanz-Andrés and I. Pérez-Grande
}

\begin{abstract}
Lift and velocity circulation around airfoils are two aspects of the same phenomenon when airfoils are not stalled and the Kutta-Joukowski theorem applies. This theorem establishes a linear dependence between lift and circulation, which breaks when stalling occurs. As the angle of attack increases beyond this point, the circulation vanishes. Since the circulation determines to a great extent the position of the forward stagnation point on an airfoil, the measurement of this position is an easy and simple way to determine the circulation, which is of help in understanding the role of the latter in the generation of aerodynamic forces on airfoils.
\end{abstract}

\section{Introduction}

Wing profiles are aerodynamic devices which are designed to provide high values of the ratio lift/aerodynamic drag, $l / d$. Airfoils are characterised by the camber line, the thickness distribution and the angle of attack. These streamlined twodimensional bodies generally have a rounded leading edge and a wedged trailing edge, the latter being of paramount importance in generating lift.

As is well known, due to the airfoil's shape, in normal operation the flow is accelerated on the airfoil's upper side and decelerated on its lower side. Hence, the pressure decreases on the upper side and increases on the lower side, and thus a net force appears. The component of this force normal to the upstream unperturbed flow is the lift, whereas the component in the direction of the flow is the aerodynamic drag (in which viscous effects are also accounted for).

Although viscosity must be considered to explain lift, in normal flight conditions viscosity effects are much smaller than inertial ones (which means high values of the Reynolds number), and they are limited to both the upper side and the lower side boundary layers that develop at the forward stagnation point, close to the leading edge. Since in many practical situations normal pressure gradients are negligible in these boundary layers [1], viscosity effects can be neglected when pressure forces acting on the airfoil surfaces are calculated. This explains why aerodynamic potential models are so accurate in predicting pressure forces on airfoils when the boundary layer is not separated.

A measure of the flow acceleration and deceleration on the airfoil surface is the circulation, $\Gamma$, defined as the integral of the flow velocity, $\mathbf{V}$, on the airfoil surface:

$$
\Gamma=\oint \mathbf{V} \cdot \mathrm{d} \mathbf{l}
$$


Within the frame of incompressible potential flow the circulation is related to the lift, $l$, through the well known Kutta-Joukowski theorem, $l=\rho_{\infty} U_{\infty} \Gamma$, where $\rho_{\infty}$ and $U_{\infty}$ are the fluid density and the fluid velocity far upstream from the airfoil, respectively [2].

In aerodynamics, instead of the aerodynamic forces, the lift coefficient and the drag coefficient are used:

$$
\left.\begin{array}{c}
c_{l}=\frac{l}{\frac{1}{2} \rho_{\infty} U_{\infty}^{2} c} \\
c_{d}=\frac{d}{\frac{1}{2} \rho_{\infty} U_{\infty}^{2} c}
\end{array}\right\}
$$

where $c$ is the airfoil chord. For a wide range of angles, the lift coefficient varies linearly or almost linearly with the angle of attack. Then, assuming the airfoil is not equipped with any leading-edge or trailing-edge high-lift devices (like slats or flaps), since in normal flight conditions the airfoil geometry (camber and thickness) is fixed, and assuming the flight speed is also fixed, the only way to increase the lift coefficient (hence the circulation) is to increase the angle of attack. However, this cannot be done indefinitely because, at high angles of attack, separation of the boundary layer at the airfoil's upper side takes place and the airfoil stalls. Once stalling occurs, the lift, hence the circulation, ceases to grow, and beyond this point any further increase in the angle of attack leads to both a decrease in lift and an increase in aerodynamic drag.

When the angle of attack increases, a suction pressure peak is created on the upper surface near the airfoil's leading edge, and the adverse pressure gradient behind this peak may result in boundary layer separation and stall. The boundary layer, which (as stated above) starts to develop at the forward stagnation point, is laminar when it reaches the pressure peak. Therefore, behind the suction peak any one of the four following types of flow may be obtained:

(1) laminar separation without subsequent reattachment;

(2) laminar separation with immediate reattachment or with reattachment after a certain distance;

(3) transition from a laminar to a turbulent boundary layer, with subsequent separation;

(4) transition from a laminar to a turbulent boundary layer, without subsequent separation.

Following McCullough and Gault [3], three basic types of stall may occur, depending on the maximum dimensionless thickness of the airfoil (the ratio of the maximum airfoil thickness to the chord, $T$ ) and the Reynolds number (see also $[4,5])$, namely:

(1) Stall through trailing edge separation on thick profiles $(T>0.15)$. The boundary layer has become turbulent and separates near the trailing edge. With 
increasing angle of attack, the separation point moves forward, and the slope of the lift curve decreases gradually until maximum lift is obtained.

(2) Stall through leading edge separation followed by flow reattachment (short bubble) on medium-thick profiles $(0.08<T<0.15)$. At a certain angle of attack, the immediate reattachment of the separated laminar boundary layer (short bubble) ceases and complete disruption of the flow suddenly occurs over the entire upper surface of the airfoil. The plot of lift against angle of attack is linear up to the maximum lift.

(3) Stall of thin profiles $(T \leq 0.08)$. At a certain angle of attack, laminar separation is obtained, but after some distance the flow reattaches (long bubble). As the angle of attack is increased, the point of reattachment moves gradually towards the trailing edge, whereby the lift curve deflects to a maximum value which is lower than for the other cases.

Whatever the type of stall, stalling means a drastic decrease in circulation. Once separation of the upper boundary layer takes place, the Kutta-Joukowski theorem, $l=\rho_{\infty} U_{\infty} \Gamma$, is no longer valid and the circulation decreases quickly. However, the lift coefficient is still high due to the difference in pressures between both sides of the airfoil (where the boundary layer is detached, pressure coefficients are negative, whereas at the airfoil's lower side there is a stagnation point). In this configuration both lift and drag are of the same order and the airfoil's efficiency, $l / d$, is very low.

According to potential-flow models, the airfoil circulation is determined by the so-called Kutta condition at the trailing edge, which in turn fixes the position of the forward stagnation point. As the angle of attack increases, the forward stagnation point moves smoothly away from the leading edge. When the airfoil is not stalled, the stagnation point remains close to the leading edge, its position being accurately predicted by models. Once stalling occurs (at high values of angle of attack) models fail, but even in such a case reasonable analytical predictions can be obtained, provided the appropriate value of the circulation is set. Therefore, the position of the forward stagnation point is a simple and easily observable measure of the effect of circulation on the airfoil's aerodynamics.

In the following sections some simple analytical models of the position of the leading edge's stagnation point, both with and without circulation, are presented. The experimental apparatus used to check theoretical results is described, and measured experimental results corresponding to three different profiles (Fig. 1) are presented and analyzed. Finally, conclusions are outlined.

\section{Analytical background}

To analyze the influence of the circulation on the position of the forward stagnation point, let us consider a biconvex airfoil formed by two arcs of circle, as sketched in Fig. 1. Assuming potential flow, the problem can be solved by using the classical tool of conformal mapping, by transforming the contour of the airfoil into that of a circle. The Kármán-Trefftz-like conformal transformation: 


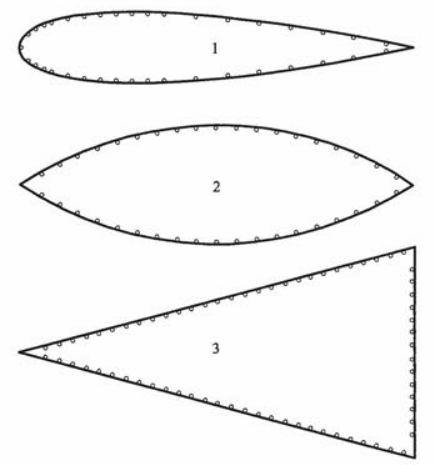

Fig. 1 Sketches of the different tested airfoils: (1) NACA 0018 airfoil, (2) biconvex airfoil, and (3) isosceles triangular profile. Circles on the profiles indicate the position of the pressure taps.

$$
\left(\frac{t+1}{t-1}\right)^{\frac{1}{\gamma}}=\frac{\tau+1}{\tau-1}
$$

with $\gamma=2(\pi-\delta) / \pi$ maps a biconvex airfoil in the complex $t$-plane onto a circle in the complex $\tau$-plane (to write the above expression, it has been assumed that all lengths have been made dimensionless, by relating them to half the airfoil chord, $c / 2$ ). When $\tau \rightarrow \infty$, the variable $t$ behaves as:

$$
t=\frac{\left(\frac{\tau+1}{\tau-1}\right)^{\gamma}+1}{\left(\frac{\tau+1}{\tau-1}\right)^{\gamma}-1}=\frac{\left(1+\frac{1}{\tau}\right)^{\gamma}+\left(1-\frac{1}{\tau}\right)^{\gamma}}{\left(1+\frac{1}{\tau}\right)^{\gamma}-\left(1-\frac{1}{\tau}\right)^{\gamma}}=\frac{1+O\left(\frac{1}{\tau^{2}}\right)}{\frac{\gamma}{\tau}+O\left(\frac{1}{\tau^{3}}\right)}=\frac{1}{\gamma} \tau+O\left(\frac{1}{\tau^{2}}\right)
$$

From this last expression it may be deduced that the uniform flow of velocity $U_{\infty}$ in the $\tau$-plane (whose direction is at angle $\alpha$ to the horizontal axis) is transformed into a uniform flow with the same angle of attack, $\alpha$, in the $t$-plane, but with intensity $U_{\infty} / \gamma\left(\right.$ instead of $\left.U_{\infty}\right)$.

The complex potential of the problem defined in the $t$-plane is the sum of the complex potentials of a uniform flow, a doublet placed at the origin of coordinates and a vortex of the appropriate intensity, also at the origin of coordinates:

$$
f(\tau)=\frac{1}{\gamma} U_{\infty}\left(\mathrm{e}^{-i \alpha} \tau+\frac{\mathrm{e}^{i \alpha}}{\tau}+\frac{i \gamma \Gamma}{2 \pi U_{\infty}} \ln \tau\right)
$$

At the stagnation points, the complex velocity vanishes:

$$
\frac{\mathrm{d} f(\tau)}{\mathrm{d} \tau}=\frac{1}{\gamma} U_{\infty}\left(\mathrm{e}^{-i \alpha}-\frac{\mathrm{e}^{i \alpha}}{\tau^{2}}+\frac{i \gamma \Gamma}{2 \pi U_{\infty}} \frac{1}{\tau}\right)=0
$$

and from this equation the value of the circulation, $\Gamma$, is obtained once the Kutta condition for the airfoil's trailing edge is imposed (the transformed trailing edge of the airfoil on the circumference must be a stagnation point on the circle). Then, 


$$
\frac{i \gamma \Gamma}{2 \pi U_{\infty}}=\mathrm{e}^{i \alpha}-\mathrm{e}^{-i \alpha}=2 i \sin \alpha
$$

After the introduction of this value of the circulation in equation 7 , the equation for the stagnation points becomes $\exp (2 i \theta)+[\exp (2 i \alpha)-1] \exp (i \theta)-\exp (2 i \alpha)=0$, whose roots are $\theta=0$ and $\theta=\pi+2 \alpha$. Note that if the circulation is cancelled, $\Gamma=$ 0 , the roots giving the positions of the stagnation points on the circle are $\theta=\alpha$ and $\theta=\pi+\alpha$. Hence the stagnation point close to the leading edge can be expressed as $\theta=\pi+k \alpha$, with $k=1$ when $\Gamma=0$ and $k=2$ when $\Gamma \neq 0$.

As the forward stagnation point on the circumference lies on a regular point of the conformal transformation, the forward stagnation point on the airfoil directly results from equation 3 . Taking into account that $\tau_{\mathrm{S}}=\exp [i(\pi+k \alpha)]=-\exp (i k \alpha)$, and that $i^{\gamma}=-\exp (-i \delta)$, after some manipulation it is possible to obtain:

$$
t_{\mathrm{S}}=\frac{H \cos \delta-1-i H \sin \delta}{H \cos \delta+1-i H \sin \delta}
$$

where $H=(\tan k \alpha)^{\gamma}$. Finally, the real part of $t_{\mathrm{S}}$ gives the dimensionless position of the forward stagnation point on the airfoil chord, $x_{\mathrm{S}}$, that is:

$$
x_{\mathrm{S}}=\frac{H^{2}-1}{H^{2}+1+2 H \cos \delta}
$$

It must be pointed out that in dimensionless variables the airfoil chord spans from -1 to 1 , thus results should be rescaled to refer them to a more familiar interval, $\left[-\frac{1}{2}, \frac{1}{2}\right]$ or $[0,1]$, for example.

The wedged leading edge of the biconvex airfoil, in a real flow, forces the boundary layer detachment at the leading edge irrespective of the value of the angle of attack, provided that this angle is high enough. It must be also pointed out that the potential solution without circulation is absolutely unrealistic at the trailing edge: in the potential solution a rear stagnation point appears at the airfoil's upper side.

To analyze the effect of a rounded leading edge, the well known Joukowsky transformation can be used. The Joukowski transformation, $t=\tau+1 / \tau$, maps a circumference centred in $\tau_{0}=-\lambda$ and radius $1+\lambda$ onto a symmetric Joukowski airfoil spanning in the $t$-plane from $-(1+2 \lambda)-1 /(1+2 \lambda)$ to $2, \lambda$ being a dimensionless parameter related to the airfoil's dimensionless thickness, $T$, through the expression $T=0.961 \lambda^{2}+0.729 \lambda$.

Taking into account the above reasoning on the stagnation point on the circumference (equations 5-7 are the same), the forward stagnation point (the one defined by the root $\theta=\pi+k \alpha$ ) is now:

$$
t_{\mathrm{S}}=-[\lambda+(1+\lambda) \exp (i k \alpha)]
$$

whose real part is:

$$
x_{\mathrm{S}}=-[\lambda+(1+\lambda) \cos k \alpha]\left(1+\frac{1}{\lambda^{2}+(1+\lambda)^{2}+2 \lambda(1+\lambda) \cos k \alpha}\right)
$$


Obviously, the same comments on the convenience of rescaling the results to a normalised dimensionless chord length already introduced for a biconvex airfoil apply to this case. Note that equation 11 with $\lambda=0$ corresponds to a flat plate defined in $[-2,2]$, the forward stagnation point being $x_{\mathrm{S}}=-2 \cos (k \alpha)$. Obviously, for small values of the angle of attack the position of the forward stagnation point behaves as $x_{\mathrm{S}}=-2+(k \alpha)^{2}$. The same results can be obtained from equation 9, taking $\delta=0$ (which means $\gamma=0$ ), although in this case, since analytical results are referred to the interval $[-1,1]$, the resulting expression for the position of the forward stagnation point is $x_{\mathrm{S}}=-\cos (k \alpha)$. Obviously, the same comment already made for the biconvex airfoil concerning the rear stagnation point applies here.

Analytical results are presented in Fig. 2, where the variation with the angle of attack of the position of the forward stagnation point for different airfoils, either with circulation or without circulation, are plotted (in the same graph experimental results are also presented). As can be seen, for the same value of the angle of attack, the position of the stagnation point is quite different, depending on the value of the
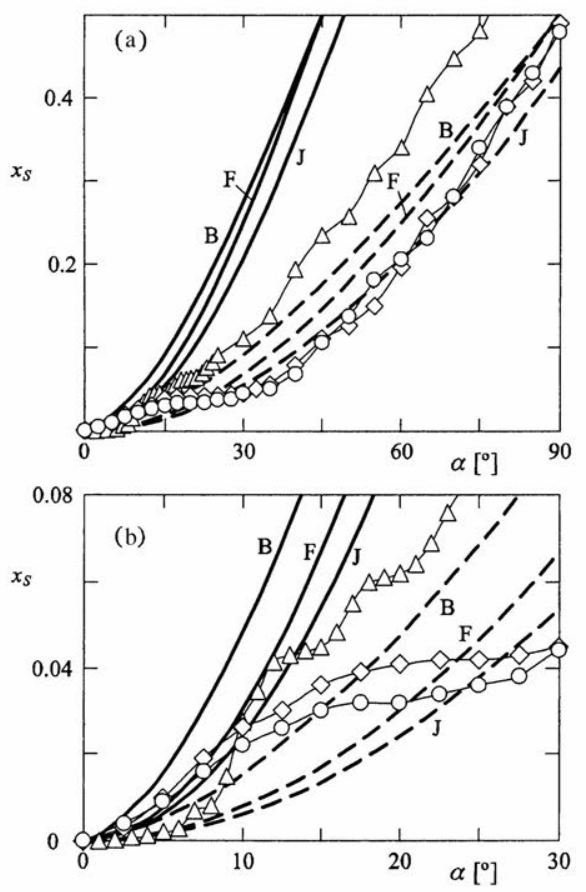

Fig. 2 Variation with the angle of attack, $\alpha$, of the dimensionless position of the forward stagnation point, $\mathrm{x}_{S}$, for different airfoils: (a) the whole range $0^{\circ} \leq \alpha \leq 90^{\circ}$; (b) details close to $\alpha=0^{\circ}$. Line type indicates potential result with circulation (solid) and without circulation (dashed) and the labels on the lines indicate the airfoil type: biconvex (B), flat plate $(F)$ and Joukowski $(J)$. Symbols identify experimental results according to the following key: NACA 0018 airfoil (circles), biconvex airfoil (rhombi), and isosceles triangular profile (triangles). 
circulation, $\Gamma$, this factor being even more important than the airfoil's shape. According to this plot, one can expect that, for small values of the angle of attack, the position of the stagnation point will be similar to the theoretical predictions for $\Gamma \neq 0$, but for large values of $\alpha$ (once the airfoil has stalled) the real behaviour should be close to the theoretical predictions for $\Gamma=0$. Hence, the position of the forward stagnation point (which can be easily measured with non-sophisticated instrumentation) gives a good qualitative description of the properties of the flow past the airfoil.

\section{Apparatus and experimental results}

An open-circuit, home-made, closed-test-chamber wind tunnel was used for the experiments reported in this paper. The wind tunnel test chamber is $0.15 \mathrm{~m}$ wide, $0.80 \mathrm{~m}$ high and $1.20 \mathrm{~m}$ long. The wind velocity profile at the model test section is uniform within $\pm 1 \%$, the turbulence intensity being around $4 \%$. The wind velocity of the stream at the test section of the wind tunnel is up to $30 \mathrm{~m} \cdot \mathrm{s}^{-1}$, which provides Reynolds numbers higher than $4 \times 10^{5}$, based on the tested model chords $(\mathrm{c} \cong 0.2 \mathrm{~m})$.

Three different airfoils were tested (Fig. 1): a NACA 0018 airfoil (without camber and a relative thickness $T=0.18$ ), a biconvex airfoil with a relative thickness $T=$ 0.3 , and an isosceles triangular profile with a main vertex angle of $30^{\circ}$. Each airfoil was equipped with at least 39 static pressure taps installed on both the upper and the lower surfaces. Each pressure tap consisted of a brass tube, of $1 \mathrm{~mm}$ inner diameter, which was connected to the pressure measurement instrument by a plastic tube of $1 \mathrm{~mm}$ inner diameter. The plastic tubes were connected to a 48-position pressure scanner from Scanivalve Corp., equipped with a Druck PDCR22 differential pressure transducer. Transducer outputs were sampled at $20 \mathrm{~Hz}$ for 12.5 seconds for each measurement. Pressure measurements have been made dimensionless by using the values of both the static pressure and the dynamic pressure of the incident wind. Therefore, the pressure coefficient is defined, as usual, as $c_{p}=\left(p-p_{\infty}\right) / q_{\infty}$, where $p$ is the measured pressure on each tap, and $p_{\infty}$ and $q_{\infty}$ are the static and dynamic pressures upstream of the testing model, respectively.

Pressure distributions were measured for values of the angle of attack ranging from $\alpha=0^{\circ}$ to $\alpha=90^{\circ}$, in different steps, $\Delta \alpha$, between $\Delta \alpha=1^{\circ}$ and $\Delta \alpha=5^{\circ}$ (depending on the region of angles of attack under consideration). From the measured pressure distributions, the position of the forward stagnation point (the dimensionless distance along the chord from the leading edge to the point where $c_{\mathrm{p}}=1$ ) as well as the aerodynamic force coefficients (both lift and drag coefficients) were determined.

Experimental results in the whole range $0^{\circ} \leq \alpha \leq 90^{\circ}$ are shown in Fig. 2(a), and an enlarged view of the experimental results close to $\alpha=0^{\circ}$ is shown in Fig. 2(b).

The variation with the angle of attack of the lift coefficient, $c_{l}$, for the airfoils under consideration is shown in Fig. 3, and the results concerning the aerodynamic pressure drag, $c_{d p}$, are plotted in Fig. 4 . In both cases, experimental values have been corrected by using standard blockage correction methods [6] to take into account the blockage of the wind tunnel test chamber due to the model under testing. On the same plots, results from another source [7], measured at Reynolds numbers much 


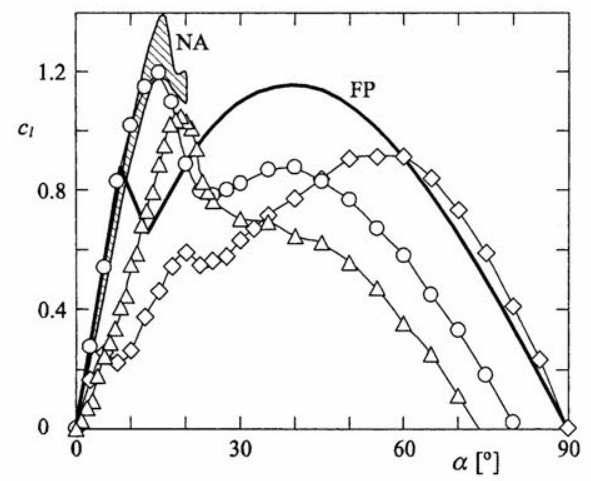

Fig. 3 Variation with the angle of attack, $\alpha$, of the lift coefficient, $\mathrm{c}_{b}$, of the tested airfoils. Symbols identify experimental results according to the following key: NACA 0018 airfoil (circles), biconvex airfoil (rhombi), and isosceles triangular profile (triangles). Result from Blevins [7] for a flat plate (FP) and for NACA 0018 airfoils (NA) are also included.

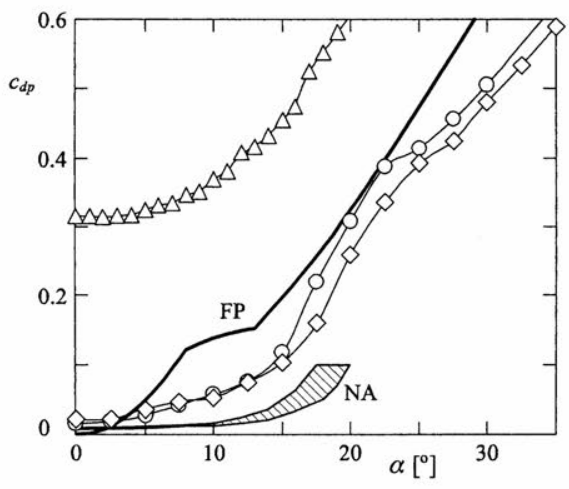

Fig. 4 Variation with the angle of attack, $\alpha$, of the pressure drag coefficient, $\mathrm{c}_{d p}$, of the tested airfoils. Symbols identify experimental results according to the following key: NACA 0018 airfoil (circles), biconvex airfoil (rhombi), and isosceles triangular profile (triangles).

Results from Blevins [7] for a flat plate (FP) and for NACA 0018 airfoils (NA) are also included.

higher than those considered here, are also shown (that fact, together with the very different testing conditions concerning both flow uniformity and turbulence level, could explain the differences on the drag coefficient of the NACA 0018 airfoil, Fig. 4).

Lift and pressure drag are defined, as usual in aerodynamics, as the component normal to the flow velocity far upstream of the model and the component in the direction of the flow velocity, respectively. According to Fig. 5, for a given value of the angle of attack the components of the pressure force in the dimensionless body axes $(\xi, \eta)$ are: 


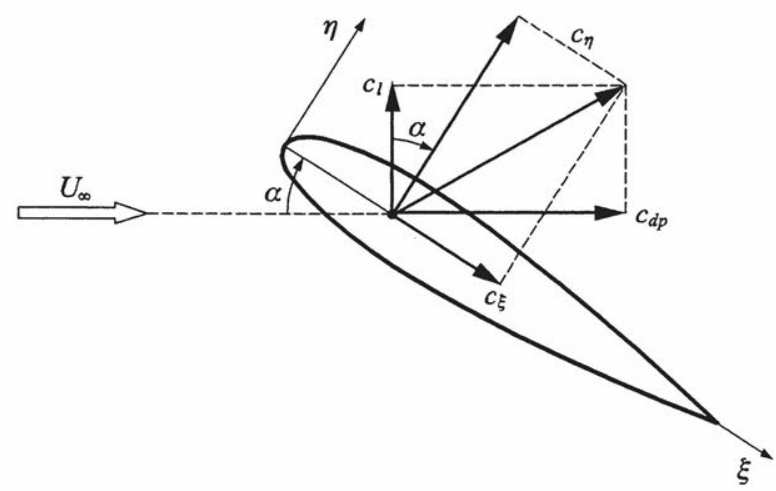

Fig. 5 Components of the aerodynamic pressure force in body axes and in wind axes.

$$
\left.\begin{array}{rl}
c_{\xi} & =\int_{0}^{1} c_{p}(\xi, \eta) \mathrm{d} \xi \\
c_{\eta} & =\int_{0}^{1} c_{p}(\xi, \eta) \mathrm{d} \eta
\end{array}\right\}
$$

Thus, the lift and pressure drag coefficients become:

$$
\left.\begin{array}{rl}
c_{l} & =c_{\eta} \cos \alpha-c_{\xi} \sin \alpha \\
c_{d p} & =c_{\eta} \sin \alpha+c_{\xi} \cos \alpha
\end{array}\right\}
$$

Obviously in the above expression for the drag coefficient only pressure forces have been considered, the total drag coefficient being $c_{d}=c_{d p}+c_{d o}$, where $c_{d o}$ is the viscous drag coefficient.

Concerning the experimental results presented in Fig. 2, in the case of airfoils with a well defined trailing edge (here the NACA 0018 and the biconvex airfoil), the forward stagnation point moves accord to predictions for $\Gamma \neq 0$ up to moderate values of the angle of attack, say $\alpha \approx 10^{\circ}$. Beyond this boundary $\left(\alpha \approx 15^{\circ}\right)$ the stagnation point excursion almost stops for a given interval of values of the angle of attack. When the angle of attack reaches large enough values $\left(\alpha \approx 20^{\circ}\right)$ the stagnation point position behaves as predicted in the $\Gamma=0$ case. Obviously, experimental and theoretical results cannot be fully compared when $\alpha$ is large, because of the very different characteristics of the two flows (real and theoretical) close to the trailing edge. However, it is remarkable that the two tested airfoils that have a well defined trailing edge (the NACA 0018 and the biconvex airfoil) behave in a similar way, in spite of their very different leading edges. The reason for such agreement resides in the existence of a sharp, wedged trailing edge, which forces the fulfilment of the Kutta condition at small angles of attack.

With the biconvex airfoil, at small angles of attack the shape of the pressure distribution is roughly similar to that of the airfoil itself, except close to the leading edge, where a stagnation point appears on the lower side of the body and a suction 
pressure peak appears on the upper side. Because of the wedge leading edge, the boundary layer separates just at that edge, although it reattaches at some distance from the edge, thus forming a recirculation bubble. As the angle of attack grows, the reattachment point moves downstream and the upper boundary layer starts to separate at the trailing edge (as happens with thick airfoils), this separation point moving upstream as the angle of attack grows. At $\alpha \approx 20^{\circ}$ the whole upper-side boundary layer is separated, and the circulation practically vanishes (however, the lift is still large for the reasons explained above, the maximum lift coefficient being reached at $\alpha \approx 60^{\circ}$ ).

The results corresponding to the triangular airfoil have been partly published elsewhere (those concerning the dependence on the angle of attack of the coefficients $c_{l}$ and $\left.c_{d p}[8]\right)$. An isosceles triangular body is very different from the shapes of standard airfoils: there is no trailing edge, and these is then a reasonable doubt about the suitability of the Kutta condition to explain lift generation. However, the triangular profile behaves like a standard-shaped airfoil provided the angle of attack is small enough, with an almost linear variation of the lift coefficient with the angle of attack. Although boundary layer separation takes place at the leading edge for very small values of the angle of attack, the shear layer reattaches on the airfoil's upper side and a recirculation bubble is formed close to the leading edge, as happens in the biconvex airfoil case. As the angle of attack grows, the reattachment point moves towards the trailing edge, so that in this range of values of the angle of attack the lift can even increase as $\alpha$ grows. This behaviour ends when the reattachment point reaches the base of the triangle $\left(\alpha \approx 18^{\circ}\right.$ ), and beyond this angle of attack reattachment is no longer possible.

For very small values of $\alpha$, the forward stagnation point keeps close to the leading edge, until a threshold value of the angle of attack is reached, as shown in Fig. 2. Such a situation corresponds to the existence of a very small recirculation bubble at the leading edge of the upper side, whose size is almost independent of $\alpha$ (the same behaviour has been reported in the case of flat plates with sharpened edges at $\alpha \approx$ $0^{\circ}$ [9]). If $\alpha$ is increased, the position of the stagnation point jumps a distance from the leading edge, analogous to the situation for airfoils with a well defined circulation, and from this point the distance grows as the angle of attack grows. This behaviour holds until the reattachment point of the recirculation bubble on the upper side reaches the base of the triangle; then, the flow separates over the whole upper surface, the lift decreases as the angle of attack increases further, and the stagnation point moves to a new curve, closer to the $\Gamma=0$ ones.

It must be remarked that, in spite of its non-aerodynamic shape, a triangular airfoil generates circulation while the recirculation bubble at the upper side (whose formation can not be avoided) remains anchored to the airfoil surface, as happens in the case of very thin standard airfoils [3-5].

\section{Conclusions}

The Kutta-Joukowski theorem stating the relationship between lift and circulation for airfoils holds for a range of angles of attack close to $\alpha=0^{\circ}$, the flow morphology 
within this range being characterised by a non-separated boundary layer. When boundary layer separation and airfoil stalling occur, the circulation starts to decrease and then vanishes, although the airfoil still produces considerable lift (as well as considerable drag, which makes the airfoil very inefficient from the aerodynamic point of view).

A simple way to visualise the effect of the airfoil circulation is by measuring the position of the forward stagnation point, since, as the angle of attack increases, drastic changes in its position take place, because of the effect of the circulation on the flow pattern over the airfoil.

\section{References}

G. K. Batchelor, An Introduction to Fluid Dynamics (Cambridge University Press, Cambridge, 1967), pp. 302-330.

L. M. Milne-Thomson, Theoretical Aerodynamics (Dover Publications, New York, 1958), pp. 91-92.

G. B. McCullough and D. E. Gault, Examples of Three Representative Types of Airfoil-Section Stall at Low Speed, NACA TN 2502 (National Advisory Committee for Aeronautics, Washington, 1951).

B. Thwaites, Incompressible Aerodynamics (Dover Publications, New York, 1960).

E. Torenbeek, Synthesis of Subsonic Airplane Design (Delft University Press and Kluwer Academic Publishers, Dordrecht, 1982).

J. B. Barlow, W. H. Rae and A. Pope, Low-Speed Wind Tunnel Testing (John Wiley \& Sons, New York, 1999), pp. 349-363.

R. D. Blevins, Applied Fluid Dynamics Handbook (Krieger Publishing Company, Malabar, 1992), pp. $322,357$.

G. Alonso, J. Meseguer and I. Pérez-Grande, 'Galloping instabilities of two-dimensional triangular cross-section bodies', Exp. Fluids, 38 (2005), 789-795.

M. J. Crompton and R. V. Barrett, 'Investigation of the separation bubble formed behind the sharp leading edge of a flat plate at incidence', Proc. Instn. Mech. Engrs. Part G, J. Aerospace Eng., 214 (2000), 157-176. 